\title{
Simultaneous Wireless Information and GaN-based Power Transfer Exploiting a Dual Frequency Band
}

\author{
J. Maximilian Placzek, \\ Peter A. Hoeher, Fellow, IEEE \\ Chair of Information and Coding Theory \\ Kiel University, 24143 Kiel, Germany \\ $\{$ jmpl,ph\}@tf.uni-kiel.de
}

\author{
Pramod K. Prasobhu ${ }^{+}$, Student Member, IEEE, \\ Marco Liserre ${ }^{+}$, Fellow, IEEE, \\ Giampaolo Buticchi*, Senior Member, IEEE \\ + Chair of Power Electronics \\ Kiel University, 24143 Kiel, Germany \\ $+\{$ pkp,ml $\} @$ tf.uni-kiel.de \\ *PEMC Group, University of Nottingham Ningbo China, \\ 315100 Ningbo, China \\ *buticchi@ieee.org
}

\begin{abstract}
A simultaneous wireless information and power transfer system employing separated frequency bands for energy and data, dubbed dual-band SWIPT, is investigated. Elementary circuit elements are optimized numerically. An experimental testbed based on a gallium nitride (GaN) full-bridge converter demonstrates data rates in excess of $450 \mathrm{kbps}$ employing onoff keying in conjunction with a simple diode detector for data recovery.

Index Terms-Inductive power transmission, Data communication, Inductive charging, Frequency division multiplexing, SWIPT
\end{abstract}

\section{INTRODUCTION}

In many applications, wireless power transfer should be coupled with data transfer - for example as a means of closed loop control of the power transfer. An elegant solution is dubbed simultaneous wireless information and power transfer (SWIPT), using a single set of coils for both functions. [1], [2]. Possible applications include medical implants and (semi)autonomous underwater apparatus, as neither plug in power/data connections nor conventional wireless communications are particularly satisfactory in such environments.

The simplest implementation of this approach is single-band SWIPT - data transfer by modulation of the power transfer. While useful for small power levels, its practicality is limited for high power levels at high efficiency. Modulation represents a random perturbation of the power transfer, disrupting optimal switching conditions and controlled operation. Furthermore, optimization of the passive components for efficient power transfer typically leads to low bandwidth and suboptimal communication. In the communications literature, fundamental information-theoretical bounds have been derived concerning the trade-off between efficiency and data rate [3]. Possible switching techniques between power and data rate have been investigated in-depth [4], [5].

This work was supported in part by the European Union/Interreg VA - Germany-Denmark, under the PE:Region Project and in part by the European Research Council under the European Unions Seventh Framework Programme (FP/2007-2013)/ERC Grant 616344-HEART and in part by the Ningbo Science \& Technology Bureau under Grant 2013A31012 and NSFC under Grant 51650110507
In order to resolve the conflicting demands with respect to bandwidth, frequency-division multiplexing (FDM) is a possible solution. FDM techniques can be realized in quite different forms. In [6], two coils are operated in parallel. Energy is transmitted via the so-called power coil, data via the so-called information coil. In the dual-band SWIPT system investigated in [7], an additional LC circuit is inserted at the primary side as well as at the secondary side. One of these additional LC circuits is employed for data transmission, the other one for data pick-up. Therefore, duplex communication is feasible. Upon properly designing the overall network, the power transfer efficiency will not significantly be decreased by the communication unit and, vice versa crosstalk will be suppressed, i.e. data communication will be reliable.

In this contribution, the dual-band SWIPT system investigated in [7] will be taken as a baseline system. However, several modifications are performed to yield improved performance: (i) The series-parallel topology favored in [7] is replaced by the series-series topology. That way, efficiency is improved when the effective load resistance is small. Also, a voltage transfer ratio close to one can be achieved for many parameter sets. (ii) In [7], a target data rate of about $20 \mathrm{kbps}$ has been achieved, which is fast enough for the control loop. In this contribution, however, a design goal is to maximize data rate given a high power efficiency. Towards these goals, all circuit components are numerically optimized. We could achieve data rates of $450 \mathrm{kbps}$ and beyond without using a complicated data equalizer or compromising power transfer. (iii) Last but not least, we attempted to utilize GaN HEMTs in an ongoing effort to exceed Si MOSFET performance. Besides computer-based component optimization, experimental results have been obtained.

\section{SySTEM OVERVIEW}

\section{A. Power Transfer}

The basic schematic for a wireless power transfer system is shown in Fig. 1. A grid front-end (active or passive) supplies a DC link, then a DC/AC converter feeds the primary coil, that acts as a transformer with the secondary coil. An AC/DC 
stage in the secondary side performs the final conversion stage and feeds the battery/load. The most adopted solution for coil excitation is to use a power electronics converter to generate a voltage square wave on the primary coil [8]. Although capacitors may be used for compensation, some authors propose more complex matching networks to improve transmission gain over wider coupling range [9].

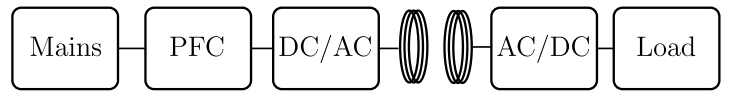

Fig. 1: Basic schematic of a wireless power transfer system.

We choose the series-series topology as the most common and practical approach from literature.

The voltage gain depends on the distance of the coils, hence the coupling factor, the effective load resistance, and the operating frequency. Having primary converter control with near resonant frequency operation for regulation can lead to lower efficiency despite reduced number of processing stages [10]. To add another degree of freedom, voltage can be controlled via a primary and/or secondary DC/DC converter or specialized modulation schemes of the main DC/AC converter, i.e. asymmetric PWM, enabling resonant switching in one leg while using the other for modulation.

The schematic topology of the overall system under investigation is shown in Fig. 2. At the primary side, a full-bridge DC-AC converter is implemented. Voltage and inner resistance of the source are denoted as $V_{s}$ and $R_{s}$. The power source drives a series circuit $C_{1}, L_{1}$. This circuit is in resonance with the series circuit $C_{2}, L_{2}$ on the secondary side. The coupling coefficient is defined as $k_{12}=M_{12} / \sqrt{L_{1} L_{2}}$, where $M_{12}$ is the mutual inductance between the power coils $L_{1}$ and $L_{2}$. Compensation is performed such that $f_{1}=1 /\left(2 \pi \sqrt{\left(L_{1}+L_{3}\right) C_{1}}\right)=$ $1 /\left(2 \pi \sqrt{\left(L_{2}+L_{5}\right) C_{2}}\right)$ is the resonant frequency for power transfer (neglecting bifurcation), where $L_{3} \ll L_{1}$ and $L_{5} \ll L_{2}$. The ac resistances of $L_{1}$ and $L_{2}$ are denoted as $R_{1}$ and $R_{2}$, respectively. At the power pick-up side, a full-bridge rectifier is implemented. The load resistance $R_{L}$ is varied in order to emulate different output powers, charging states, and battery types.

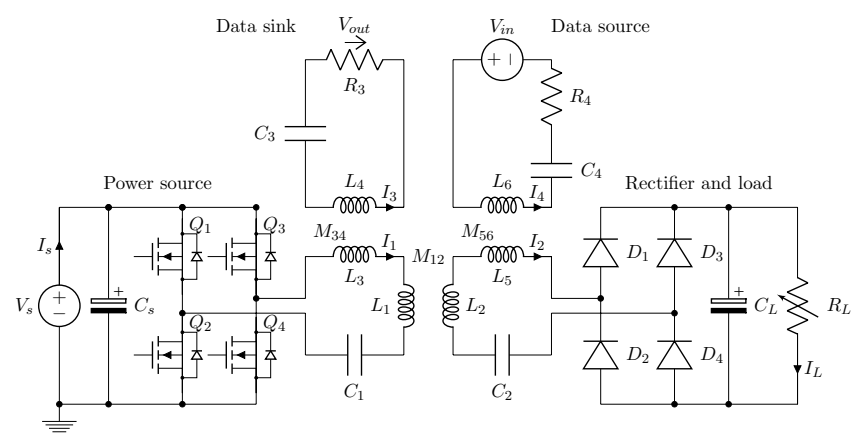

Fig. 2: Topology of the overall system under investigation.

\section{B. Data Transfer}

As suggested in [7], transformers consisting of coils $L_{3}, L_{4}$ and $L_{5}, L_{6}$, respectively, are inserted into the main loop for the purpose of data transmission, subsequently dubbed data coils. The ratios $L_{3} / L_{4}$ and $L_{5} / L_{6}$ are proportional to the turns ratio, which is assumed to be one. The corresponding mutual inductances are denoted as $M_{34}$ and $M_{56}$, consequently $k_{34}=M_{34} / \sqrt{L_{3} L_{4}}$ and $k_{56}=M_{56} / \sqrt{L_{5} L_{6}}$. Both transformers are part of LRC networks $L_{4}, R_{3}, C_{3}$ and $L_{6}, R_{4}, C_{4}$, respectively. The resonant frequency of these networks is $f_{2}=$ $1 /\left(2 \pi \sqrt{L_{4}^{\prime} C_{3}}\right)=1 /\left(2 \pi \sqrt{L_{6}^{\prime} C_{4}}\right)$, where $L_{4}^{\prime}$ is an equivalent inductance approximated as $1 / L_{4}^{\prime} \approx 1 / L_{4}+1 / L_{1} \approx 1 / L_{4}$. Similarly, $1 / L_{6}^{\prime} \approx 1 / L_{6}+1 / L_{2} \approx 1 / L_{6}$. The resonant frequency $f_{2}$ should be at least about one decade higher than the resonant frequency $f_{1}$ for power transfer in order to suppress crosstalk. The voltage of the data source is called $V_{i n}$. Obviously there is a trade-off between power efficiency and data reliability, which can be controlled by the ratio $V_{s} / V_{i n}$. Resistor $R_{4}$ is subject for optimization. It includes the inner resistance of the data source and the ac resistance of $L_{6}$. The data output is the voltage drop across resistor $R_{3}$, which includes the ac resistance of $L_{4}$. Resistor $R_{3}$ is subject for optimization as well, along with all capacitors and inductors addressed so far. Since our main concern is on data transmission via the return link, the Tx unit (data source) is inserted at the secondary side, whereas the Rx unit (data sink, data pick-up) is inserted at the primary side. However, system analysis does not change when Tx and Rx unit would be exchanged, providing that the two frequency bands are sufficiently splitted. Even full duplex communication is feasible. For symmetry reasons, we assume a series circuit in both data loops, whereas in [7] a parallel circuit has been taken at the Rx unit.

\section{System ANALYSIS}

Tasks and constraints of system optimization are manifold:

- Of primary concern is to maximize efficiency $\eta$ of power transfer.

- Simultaneously, data rate should be optimized. Since data rate depends on many parameters, including bandwidth, signal-to-noise ratio, modulation scheme, data detection, equalization, channel estimation and so forth, focus will be here on maximizing the channel capacity. Channel capacity is the maximum data rate at which information can be transmitted virtually error free.

- Finally, the power transfer efficiency shall not significantly be decreased by the communication unit and, vice versa crosstalk shall be suppressed, i.e. data communication shall be reliable.

The free parameters to be optimized include $C_{1} \ldots C_{4}, L_{1} \ldots L_{6}$ and $R_{3}, R_{4}$, as well as voltages $V_{s}$ and $V_{i n}$. In order to obtain an overall optimization at reasonable computational complexity, we propose to start with a maximization of $\eta=P_{2} / P_{1}$ given inactive data loops $\left(R_{3}=R_{4} \rightarrow \infty, P_{\text {data }}=0\right)$, and to freeze these parameters. Afterwards, channel capacity will be optimized, given several constraints. This two-step design procedure is not conflicting as verified numerically. 


\section{A. Kirchhoff's Circuit Laws}

The overall system can completely be expressed by Kirchhoff circuit laws

$$
\begin{aligned}
& V_{R_{s}}(s)+V_{L_{3}}(s)+V_{C_{1}}(s)+V_{R_{1}}(s)+V_{L_{1}}(s)= V_{s}(s) \\
&-V_{L_{2}}(s)+V_{R_{2}}(s)+V_{C_{2}}(s)+V_{L_{5}}(s)+V_{R_{L}}(s)=0=0 \\
& V_{C_{3}}(s)+V_{L_{4}}(s)-V_{\text {out }}(s)=0 \\
& V_{R_{4}}(s)+V_{C_{4}}(s)+V_{L_{6}}(s)=V_{\text {in }}(s) \\
&-V_{L_{1}}(s)+s L_{1} I_{1}(s)-s M_{12} I_{2}(s)=0 \\
&-V_{L_{2}}(s)-s L_{2} I_{2}(s)+s M_{12} I_{1}(s)=0 \\
&-V_{L_{3}}(s)+s L_{3} I_{1}(s)+s M_{34} I_{3}(s)=0 \\
&-V_{L_{4}}(s)+s L_{4} I_{3}(s)+s M_{34} I_{1}(s)=0 \\
&-V_{L_{5}}(s)+s L_{5} I_{2}(s)+s M_{56} I_{4}(s)=0 \\
&-V_{L_{6}}(s)+s L_{6} I_{4}(s)+s M_{56} I_{2}(s)=0
\end{aligned}
$$

together with ten component laws of the form

$$
\begin{aligned}
-V_{R_{i}}(s)+I_{i}(s) R_{i} & =0 \\
-V_{C_{i}}(s)+I_{i}(s) /\left(s C_{i}\right) & =0 .
\end{aligned}
$$

These equations can be expressed in vector/matrix form as:

$$
\mathbf{A} \cdot \mathbf{x}=\mathbf{b}
$$

Consequently, the unknowns in $\mathbf{x}$ are obtained as

$$
\mathbf{x}=\mathbf{A}^{-1} \cdot \mathbf{b} .
$$

Frequency splitting [11], also called bifurcation, is inherently included in the formulas at short distances between the power coils.

\section{B. Results for Power Transfer Unit}

In the prototype under investigation, power coils with an inductance of $L_{1}=L_{2}=16.8 \mu \mathrm{H}$ are used. The ac resistance of $R_{1}$ and $R_{2}$ is experimentally obtained as $R_{1}(f)=$ $R_{2}(f) \approx R_{D C}\left(1+9.42 \cdot 10^{-10} \cdot f^{2}\right)$ with $R_{D C}=12.7 \mathrm{~m} \Omega$. This approximation is quite tight for frequencies up to about $150 \mathrm{kHz} . L_{3}=L_{4}$ is initially chosen to be $0.1 L_{1}$ (and optimized subsequently). All these parameters are fixed throughout the optimization of the power transfer unit.

Assuming $V_{L}=24 \mathrm{~V}$ without loss of generality, the effective load resistance is $R_{L}=5.76 \Omega, 2.88 \Omega, 1.152 \Omega$, and $0.576 \Omega$ at an output power of $100 \mathrm{~W}, 200 \mathrm{~W}, 500 \mathrm{~W}$, and $1 \mathrm{~kW}$, respectively. As a default number, $P_{2}=200 \mathrm{~W}$ (i.e., $R_{L}=$ $2.88 \Omega$ ) is taken.

For symmetry reasons, in numerical results we assume $C_{1}=$ $C_{2}$ since $L_{1}=L_{2}$. In that case optimization simplifies for $L_{3}=$ $L_{4}=L_{5}=L_{6}$ and $C_{3}=C_{4}$. The default coupling coefficient of the power loop is $k_{12}=0.5$, which corresponds to a distance of about $3 \mathrm{~cm}$.

In order to reduce the number of parameters when optimizing the efficiency of the power loop, $\eta=P_{2} / P_{1}$, the data loops are deactivated. Towards this goal, $R_{3}=R_{4} \rightarrow \infty$. The remaining parameters are $R_{L}$ and $k_{12}$, if $\eta$ is plotted versus resonant frequency $f_{1} . C_{1}=C_{2}$ are determined as

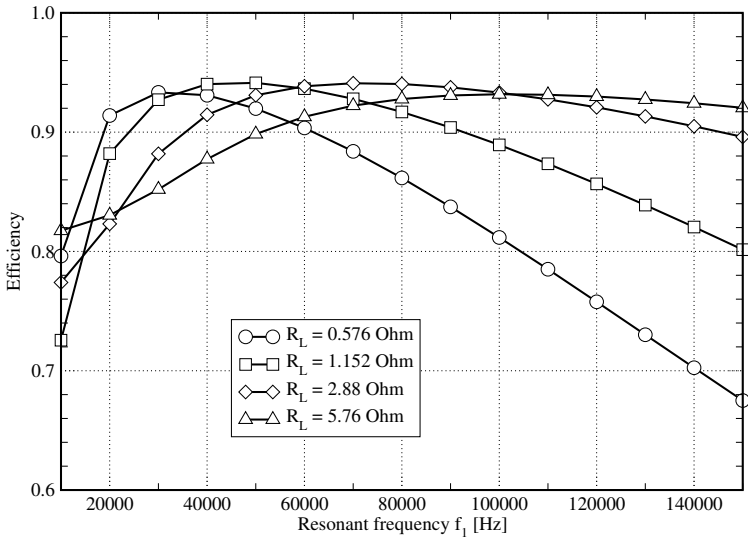

Fig. 3: Efficiency as a function of resonant frequency $f_{1}$ given coupling coefficient $k_{12}=0.5$.

$C_{1}=1 /\left[\left(L_{1}+L_{3}\right) \cdot\left(2 \pi f_{1}\right)^{2}\right]$, where $f_{1}$ is changed between $10 \mathrm{kHz}$ and $150 \mathrm{kHz}$.

As shown in Fig. 3, the resonant frequency should be carefully selected in order to maximize efficiency. At $R_{L}=$ $2.88 \Omega$, the optimum frequency is $f_{1} \approx 70 \mathrm{kHz}$. Upon reducing/increasing $R_{L}$, the optimum $f_{1}$ reduces/increases. When reducing/increasing the coupling coefficient $k_{12}, f_{1}$ should be increased/decreased. Subsequently, $f_{1}$ is fixed to be $70 \mathrm{kHz}$ at default parameters $R_{L}=2.88 \Omega$ and $k_{12}=0.5$. When activating both data loops, the same optima are obtained as long as $f_{2} \gg f_{1}$ and $V_{i n} / V_{s}$ is reasonably small. In other words: The data loops do not affect the optimization of the resonant frequency of power transfer.

\section{Results for Data Transfer Unit}

Since a periodic square-wave source signal $v_{s}(t)$ generates harmonics at odd multiples of $f_{1}$ and causes minima at even multiples of $f_{1}$, a favorite design rule is

$$
f_{2}=2 n \cdot f_{1},
$$

where $n$ is a positive integer number.

Let us divide the frequency range into frequency bins of bandwidth $\Delta B$ each. Channel capacity of the data unit is defined as [12]

$$
C=\lim _{\Delta B \rightarrow 0} \frac{\Delta B}{2} \sum_{i} \log _{2}\left(1+S N R_{i}\right),
$$

where $S N R_{i}=P_{i} / N_{i}$ is the signal/noise ratio of the $i$ th frequency bin. In the system under investigation, the lowest frequency bin is determined by $f_{1}$ (because of the crosstalk constraint $f_{2}>10 f_{1}$ ), whereas the largest frequency bin is determined by the self resonance of the power coil. In order to get rid of such a fussy definition, in our numerical results we take only those frequency bins into account that are subject to $S N R_{i} \geq 1$, since practical receiver implementations typically fail at SNR levels below about $0 \mathrm{~dB}$. Channel capacity naturally increases with increasing resonant frequency $f_{2}$, because then more bandwidth is available. For that reason, $f_{2}$ should be as large as technically possible. Since self resonance is at 


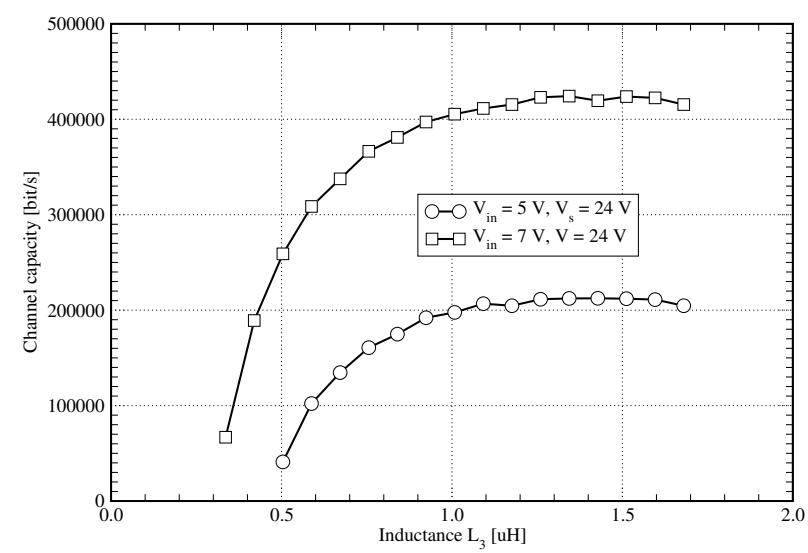

Fig. 4: Channel capacity as a function of $L_{3}$ given the default parameters under investigation.

about $6.1 \mathrm{MHz}$ for the power coils used in the prototype, the region up to $4.5 \mathrm{MHz}$ is best suitable for $f_{2}$. Therefore, we select $f_{2}=4.48 \mathrm{MHz}=64 f_{1}$.

Channel capacity is found to be independent of $R_{3}$. Without loss of generality, we choose $R_{3}=10 \Omega$. With increasing $R_{3}$, current $I_{3}$ reduces, but noise is likely to become more dominant.

Channel capacity monotonically improves with decreasing resistance $R_{4}$. There is a trade-off between the quality factor $Q$ and the length of the impulse response. We select $R_{4}=1 \Omega$, because for $R_{4}<1 \Omega$ the impulse response would be very long.

Since $f_{1}, f_{2}$, and all parameters of the power loop are already optimized, and since $R_{3}$ and $R_{4}$ are not relevant with respect to maximizing channel capacity, the remaining parameter subject for investigation is $L_{3}=L_{4}=L_{5}=L_{6}$. Signal power $P_{i}=\operatorname{Re}\left\{V_{\text {out }, i} \cdot V_{\text {out }, i}^{*} / R_{3}\right\}$ at the data sink is obtained by solving Eq. (4) given $V_{s}=0$ and $V_{i n}>0$. Vice versa, noise power $N_{i}=\operatorname{Re}\left\{V_{\text {out }, i} \cdot V_{\text {out }, i}^{*} / R_{3}\right\}$ at the data sink is obtained by solving Eq. (4) given $V_{s}>0$ and $V_{\text {in }}=0$. Thus, comparatively the power transfer unit is the dominant noise source.

The noise power is a function of the power spectral density of the power converter. Ideally, the power converter outputs a square-wave signal in time domain with period $T=1 / f_{1}$ and amplitude $V_{s}$. The corresponding Laplace transform is

$$
V_{s}(s)=\frac{V_{s}}{s} \tanh (s T / 4)=\frac{V_{s}}{s} \frac{1-e^{s T / 2}}{1+e^{-s T / 2}},
$$

which corresponds to a line spectrum. Consequently, channel capacity is unrealistically high when the resonant frequencies are based on design rule (5). As an alternative, one may assume $V_{s}(s)=V_{s}$ for all frequencies. This case establishes the worst power spectral density. The channel capacity results reported next are based on this worst case.

As depicted in Fig. 4, channel capacity is optimized at $L_{3}=L_{4}=1.4 \mu \mathrm{H}$ for the default parameters under investigation. This value is less than $0.1 L_{1}$ and hence acceptable. The corresponding compensation capacitors are yielded as
$C_{3}=C_{4} \approx 1 /\left(L_{4}^{\prime}\left(2 \pi f_{2}\right)^{2} \approx 0.98 \mathrm{nF}\right.$. The optimal value of $L_{3}$ is independent of the ratio $V_{i n} / V_{s}$.

Channel capacity is boosted with $V_{i n}$, at the cost of effective efficiency $\eta_{\text {eff }}=P_{2} /\left(P_{1}+P_{\text {data }}\right)$. Given $V_{s}=24 \mathrm{~V}$ (about $200 \mathrm{~W} @ 2.88 \Omega), V_{i n}=5 \mathrm{~V}$ is sufficient for reliable data detection. At $V_{\text {in }}=5 \mathrm{~V}$, the impact of the data unit onto the power waveform is negligible. In the simplest case, data can be transmitted by on-off keying, i.e., $V_{\text {in }}$ is switched on or off.

\section{Practical Design Considerations}

\section{A. Power Transfer}

In case of WPT systems for EV charging, the battery charger equivalent resistance varies over a particular range. The characteristic resonant frequency shifts with varying charging port/coil distances. Accordingly, the converter operating frequency needs to be modified if the system has to be operated at optimal efficiency. Operation close to the resonant frequency minimizes reactive power and increases efficiency. In this implementation the devices are operated to achieve ZVS, hence at a frequency slightly higher than the resonant frequency. The efficiency peaks at the resonant point increases with larger coupling factors. Although the efficiency increases with the coupling factor, the gain plot in Fig. 5 shows that the achievable gain reduces at higher coupling. Hence a small trade-off with regard to the reactive power flow and desired gain needs to be considered as well.

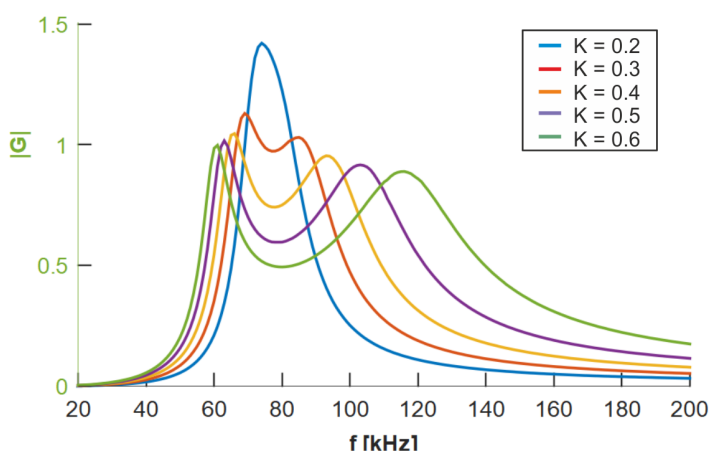

Fig. 5: Gain plots vs frequency of the wireless transfer system for various $k$ values viz - 0.2, 0.3, 0.4, 0.5, 0.6 .

Thus for a particular battery charger system, the design critical criteria are that the system should be capable of transferring the nominal power at the highest possible coupling factor (here selected as $k=0.6$ ) even at the lowest load resistance.

Using wideband gap devices like GaN further enables a highly efficient power conversion compared to Si-based solutions. This is attributed to their smaller output capacitances $C_{o s s}$ and lower drain source resistance values $R_{d s, o n}$ for similar blocking voltages and currents compared to $\mathrm{Si}$ MOSFETs which results in lower losses as shown in Fig. 6.

Analyzing the full-bridge converter operation as equivalent to an LLC resonant converter, the following design trade-offs need to be considered: 


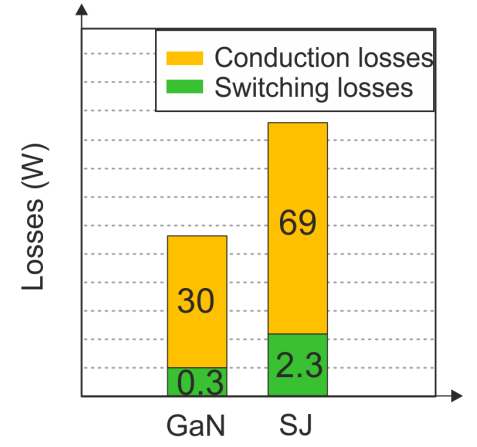

Fig. 6: Loss comparison of GaN HEMT vs Si-based Super Junction (SJ) MOSFET (GS66504B vs IPW65R190CFD).

- A smaller value of $k$ leads to a steep gain curve and hence the frequency range required to regulate converter voltage is kept small. This however causes higher circulating current. As a result, the conduction losses rise.

- A higher value of $k$ would result in a flatter curve and therefore the required frequency range to regulate the output voltage is higher. However, a higher value reduces the circulating current.

- The available magnetizing inductance has also an impact on the dead time. By increasing the magnetizing inductance, the magnetizing current is decreased and the dead time needs to be increased.

\section{B. Data Transfer}

The numerical model of the circuit describes the data coupling transformers as ideal transformers. To approximate this behavior in reality, a high coupling factor has to be achieved. Instead of utilizing conventional ferrite transformers [7], the low inductance value resulting from optimization allows the use of air-cored transformers which achieve high coupling through bifilar interlacing of separate litz conductors. The default coupling coefficient of the air-core transformers is about $k_{34}=k_{56}=0.9$ according to practical experience. For testing purposes, the data signal source $V_{i n}$ is realized as a half-bridge circuit, employing two switches and a capacitive voltage divider. A diode-based envelope detector is chosen as receiver. A pseudo-random binary sequence modulates a square wave with the fundamental frequency $f_{2}$ via on-off keying, which in turn controls the half-bridge. This setup demonstrates bandwidth, signal quality, and the possibility of processing high data rates with simple circuitry. It is, however, not necessarily the communications topology of choice for this application, a variety of modulation schemes and transceivers are viable. In this regard, the notion of signal-to-noise ratio (SNR) deserves further discussion. While the data signal is subject to relevant interference from the harmonics of the power transfer, this crosstalk is a periodic, causal, and easily predictable signal and therefore by definition not true noise. It can be compensated for by adaptive filtering, or circumvented by methods such as discrete multitone transmission (DMT) in conjunction with adaptive bit loading in order to approach Eq. (6). Adaptive bit loading implies that "noisy" subcarriers are deactivated. Similar reasoning applies to the channel impulse response. It is possible to reduce intersymbol interference by equalization.

\section{EXPERIMENTAL RESULTS}

A proof of concept of the proposed SWIPT system is realized with an experimental test-bed featuring numerically optimized passive circuit components and GaN semiconductors for the power electronics part.

\section{A. Description of Setup and Design Parameters}

The DC/DC converter implemented here is a GaN-based full-bridge with GaNSystems GS66504B on primary side driven by Silabs gate drivers controlled by microcontroller MPC5643L. The magnetic coupling system consists of litz wires and radial ferrite cores arranged behind the flat coils to improve coupling and copper utilization. The diameter of this arrangement as shown in Fig. 8 is $23 \mathrm{~cm}$. The secondary consist of MBRS10100 Si Schottky diodes in a full bridge rectifier configuration. All tests have been carried out with this configuration. The communication coupling transformers consisting of $L_{3} / L_{4}$ and $L_{5} / L_{6}$ are wound with 4 turns of a bifilar arrangement of the same litz wire used for the power coils interlaced with an additional thin conductor. Communication is driven by an IXDN630 monolithically integrated high speed half-bridge and a capacitive voltage divider, resulting in a square-wave input amplitude $V_{i n}$ to the communication system of half the auxiliary supply voltage $V_{d d}$. Data symbols are generated and received by an Analog Discovery 2 (AD), $100 \mathrm{Mbps}$ digital signal generator and oscilloscope system. The envelope detection circuit is constructed according to Fig. 7.

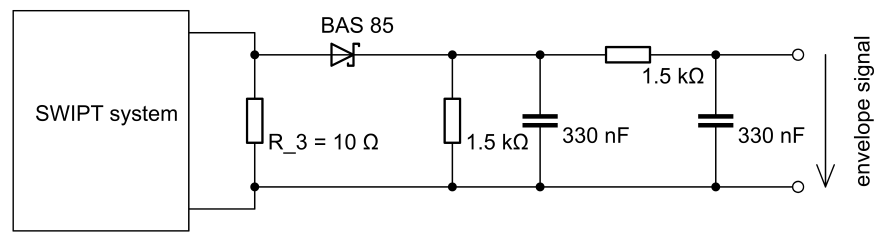

Fig. 7: Envelope detector connected to the SWIPT system.

Actual circuit parameters are tabulated as:

\begin{tabular}{|c|c||c|c|}
\hline \multicolumn{2}{|c||}{ Power System } & \multicolumn{2}{c|}{ Comm. System } \\
\hline$V_{s}$ & $15 \mathrm{~V}$ & $V_{d d}$ & $12 \mathrm{~V}$ \\
\hline$L_{1 / 2}$ & $16.8 \mu \mathrm{H}$ & $L_{3 / 4 / 5 / 6}$ & $1.2 \mu \mathrm{H}$ \\
\hline$C_{1 / 2}$ & $280 \mathrm{nF}$ & $C_{3 / 4}$ & $1 \mathrm{nF}$ \\
\hline$R_{L}$ & $2.88 \Omega$ & $R_{3}, R_{4}$ & $10 \Omega, 1 \Omega$ \\
\hline$f_{1}$ & $70 \mathrm{kHz}$ & $f_{2}$ & $3.68 \mathrm{MHz}$ \\
\hline
\end{tabular}

The measured value of $f_{2}=3.68 \mathrm{MHz}$ deviates from the optimized value reported above, most likely due to parameter deviations and system parasitics adding relevant capacity and inductance to the circuit. 


\section{B. Experimental Setup and Waveforms}

The converter system based on the above description is shown in Fig. 8. The primary GaN-based converter is placed to the right, together with the primary capacitors, the coupling transformer and the envelope detector. The two transfer coils are seen in the middle. The rectifier unit with capacitors, transformer and data transmitter are to the left. In the front is the $\mathrm{AD}$ system for communication testing.

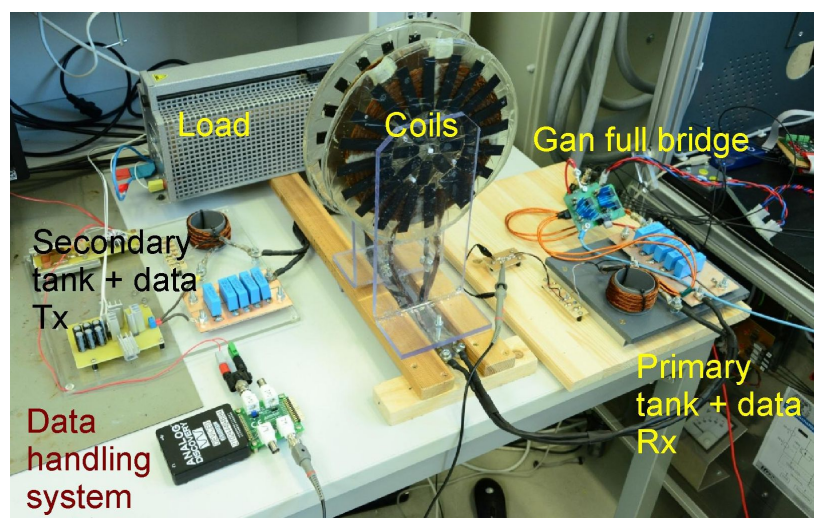

Fig. 8: GaN-based wireless information and power transfer prototype.

The measurement at particular loading current of $5 \mathrm{~A}, 15 \mathrm{~V}$ on the primary is shown in Fig. 9 given a distance of $2 \mathrm{~cm}$. The ZVS operation due to operation close to the peak frequency of $57 \mathrm{kHz}$ (due to frequency splitting) is seen in this figure. The pink blocking voltage is seen to fall to zero before the blue current changes from negative to positive ensuring ZVS.

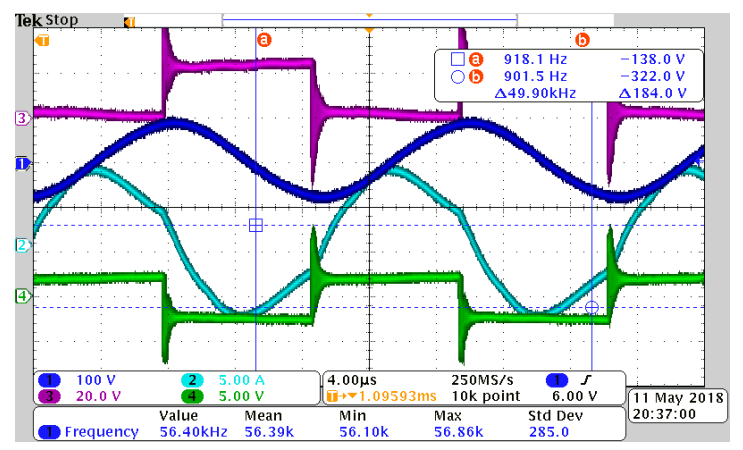

Fig. 9: Waveforms measured at the primary (blue: tank capacitor voltage, pink: drain source voltage across $\mathrm{GaN}$, blue: tank current, green: voltage at tank input).

\section{CONCLUSION}

This papers deals with a simultaneous wireless information and power transfer system employing separated frequency bands and using GaN power devices. The LRC circuit components have been numerically optimized both for power and data transfer. Although the $\mathrm{GaN}$ based converter under investigation increases the slew rate and hence adversely affects the noise spectral density, the achieved data transfer

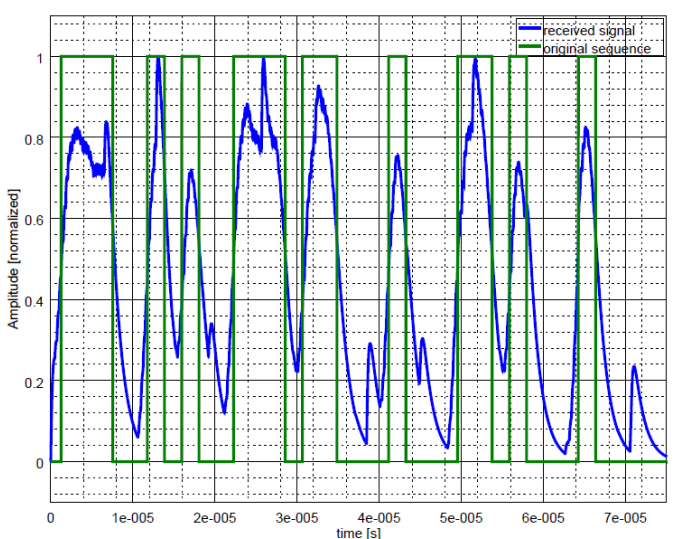

Fig. 10: Envelopes of a 32 bit pseudo-random binary sequence at a distance of $4 \mathrm{~cm}$ with power transmission running. Bit rate is $454.4 \mathrm{kbps}$. A properly selected detection threshold restores the bit sequence $[1 ; 1 ; 1 ; 0 ; 0 ; 1 ; 0 ; 1 ; 0 ; 0 ; 1 ; 1 ; 1 ; 0 ; 1 ; 1 ; 0 ; 0 ; 0 ; 1 ; 0 ; 0 ; 0 ; 1 ; 1 ; 0 ; 1 ; 0 ; 0 ; 0 ; 1 ; 0]$.

rates and the error performance demonstrate the potential of wideband gap devices for SWIPT applications.

\section{REFERENCES}

[1] S.Y.R. Hui, W. Zhong, and C.K. Lee, "A critical review of recent progress in mid-range wireless power transfer," IEEE Transactions on Power Electronics, vol. 29, no. 9, pp. 4500-4511, Sept. 2014.

[2] X. Wei, Z. Wang, and H. Dai, "A critical review of wireless power transfer via strongly coupled magnetic resonances," Energies, vol. 7, pp. 4316-4341, 2014.

[3] L. Liu, R. Zhang, and K.-C. Chua, "Wireless information and power transfer: A dynamic power splitting approach," IEEE Transactions on Communications, vol. 61, no. 9, pp. 3990-4001, Sep. 2013.

[4] I. Krikidis, S. Timotheou, S. Nikolaou, G. Zheng, D. W. K. Ng, and R. Schober, "Simultaneous wireless information and power transfer in modern communication systems," IEEE Communications Magazine, vol. 52, no. 11, pp. 104-110, Nov. 2014.

[5] S. Bi, Y. Zeng, and R. Zhang, "Wireless powered communication networks: An overview," IEEE Wireless Communications, vol. 23, no. 2, pp. 10-18, Apr. 2016

[6] T. Bieler, M. Perrottet, V. Nguyen, and Y. Perriard, "Contactless power and information transmission," IEEE Transactions on Industry Applications, vol. 38, no. 5, pp. 1266-1272, Sep./Oct. 2002.

[7] J. Wu, C. Zhao, Z. Lin, J. Du, Y. Hu, and X. He, "Wireless power and data transfer via a common inductive link using frequency division multiplexing," IEEE Transactions on Industrial Electronics, vol. 62, no. 12, pp. 7810-7820, Dec. 2015.

[8] L. Shi, Z. Yin, L. Jiang, and Y. Li, "Advances in inductively coupled power transfer technology for rail transit," CES Transactions on Electrical Machines and Systems, vol. 1, no. 4, pp. 383-396, Dec. 2017.

[9] M. Liu, S. Liu, and C. Ma, "A high-efficiency/output power and lownoise megahertz wireless power transfer system over a wide range of mutual inductance," IEEE Transactions on Microwave Theory and Techniques, vol. 65, no. 11, pp. 4317-4325, Nov. 2017.

[10] O. Knecht and J. W. Kolar, "Comparative evaluation of IPT resonant circuit topologies for wireless power supplies of implantable mechanical circulatory support systems," in Proc. IEEE Applied Power Electronics Conference and Exposition (APEC), Tampa, FL, 2017, pp. 3271-3278.

[11] A.P. Sample, D. A. Meyer, and J.R. Smith, "Analysis, experimental results, and range adaptation of magnetically coupled resonators for wireless power transfer," IEEE Transactions on Industrial Electronics, vol. 58, no. 2, pp. 544-554, Feb. 2011.

[12] T.M. Cover and J.A. Thomas, Elements of Information Theory. John Wiley \& Sons, 2nd ed., 2006. 